\title{
Overall design of Electronic Cam System based on SCM89C52
}

\author{
Wei He,Cheng Wang \\ Department of Mechanical Engineering,Nanjing Institute of Industry Technology, \\ Nanjing210046,China \\ Department of Electric-Mechanical Engineering \&AutoMation,Tianjin Vocational Institute, \\ Tianjin300410, China
}

Email: hewei1957@163.com, wangcheng198163@163.com

\begin{abstract}
Keywords:Electronic cam System; Single-chip microcomputer 89C52; Stepping motor; Electromechanical integration
\end{abstract}

\begin{abstract}
Aiming at the limitation of cam mechanism, on the basis of analyzing research actuality of electronic cam at home and abroad, electronic cam system of single-chip microcomputer(89C52)controlling stepping motor is adopted to implement straight reciprocating movement of cam mechanism follower. Furthermore, derivative process and relational expression of electronic pulse frequency is presented. Finally, advantage and application prospect of electronic cam system is discussed.
\end{abstract}

\section{Introduction}

Cam mechanism is used commonly in much mechanical equipment. It can convert rotation movement into the expected intermittent straight reciprocating movement or swung repeatedly movement, etc. But cam mechanism also exists great limitations in practice:

1) Adjustment of manufacturing and assembly error is very difficult.

2) Cam mechanism belongs to high vice point line contact and exists greater contact stress.

Therefore, we can adopt electronic cam system of single-chip microcomputer controlling stepping motor, in order to realize the motion law of the follower .An application example of electronic cam is in the packaging paper cutter,the result analyses many advantages of the impact velocity and acceleration aspect [1]. Cam mechanism can also control robot mechanical arm so as to increase the mechanical arm speed and flexibility [2]. Electronic cam can accurately realize automatic programming according to transmit distance request [3].

\section{Design of electronic cam system based on single-chip microcomputer}

In this paper, electronic cam system adopts single-chip microcomputer (89C52) as a microprocessor to control stepping motor rotation. Motor rotation through the reducer slowdown drives precision ball screw in order to convert rotation movement into slider linear reciprocating motion. Through changing the motor speed, the slider can simulate different cam contour curve. System design includes two parts of hardware design and software design.

The system hardware design.The system hardware is mainly composed of single-chip microcomputer (89C52), cam curve memory PROM (programmable read-only memory), shaft position encoder memory, D/A converter, driving amplifier, stepping motor, follower, programmable chip 8255, keyboards, LED display and clocking circuit and reset circuit, etc. Fig. 1 is the principle diagram of the system hardware. Cam displacement instruction from computer actuates displacement driver signal through $\mathrm{D} / \mathrm{A}$ converter. When the computer receives pulse coder in shaft position, it then take a corresponding displacement signal from the cam contour list stored in programmable memory PROM. Cam multiple contour can be stored in programmable memory at the same time, the corresponding cam contour can be ordered to actuator. 


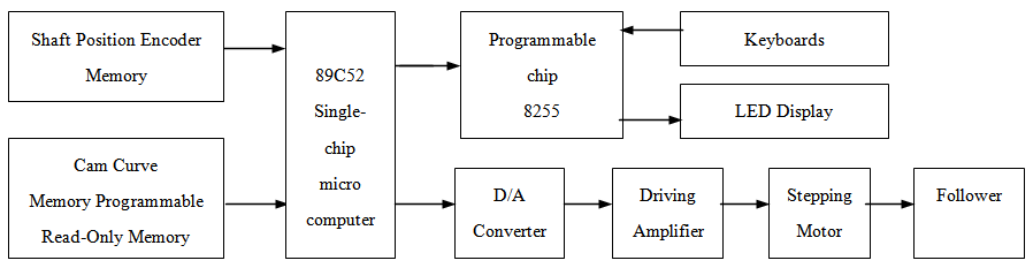

Fig. 1 Electronic cam system hardware principle diagram

The system software design.Control program of electronic cam system software includes two parts of computing main program and single-chip microcomputer control program. Firstly, computing main program is programmed to the main control dialog. Secondly, in the dialog I input motion parameters including: cam contour curve form, the highest speed of Electronic cam system follower, reduction ratio of reducer between stepping motor and screw $i$, supreme running frequency $f_{M}$ and start frequency of stepping motor $f_{0}$. Thirdly, system invokes motion parameters counting subroutine, calculates the total operation steps and gets data table of single-chip microcomputer controlling step motor. Then system invokes display subroutine and display operation parameters and prediction operation curve. Finally, system converts data table into timing initial value table of the microcontroller timer, which is written into single-chip microcomputer control program. Fig. 2 is the computing main program process of electronic cam system.

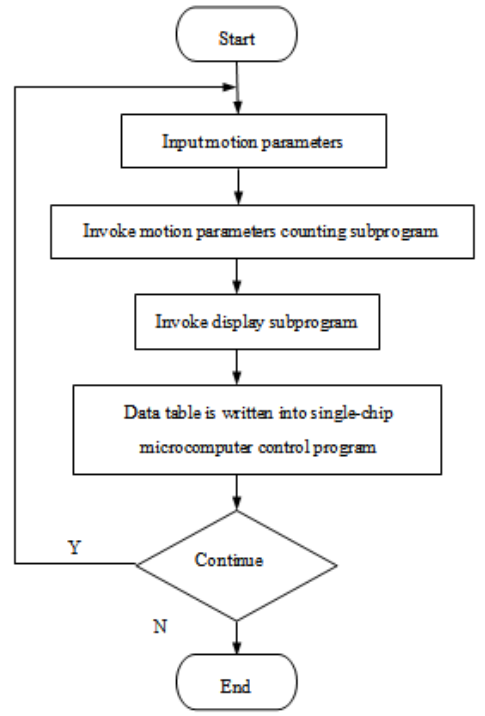

Fig.2 Computing main program processes of electronic cam system

Single-chip microcomputer control procedure mainly includes main program, disruption output control program, keyboards input program, alarm abrupt stop subprogram. When computing main program is in disruption response wait state, button disruption priority is higher than timing disruption. If it is alarming abrupt stop key, motor stops running and reinitializes the electronic cam system. If it is parameter input key, system waits for inputting parameters and confirms operation.

According to inputting cam curve form, single-chip microcomputer invokes automatically timing disruption program of corresponding cam curve and starts motor running. Each timing disruption response of single-chip microcomputer sends a driver pulse, which makes stepping motor turning an angle. Each Timing initial of disruption timer timing is collected through the look-up table subroutine in control data table. Each disruption process judges total operation step. If the value is zero, electronic cam completes a travel. If I do not press stop button, the program start next motion travel again.

If I require electronic cam system to run other cam curve of program memory storage outside the commonly used cam curve, SCM control data sheet is only rewritten.

\section{Electronic cam system electrical impulse frequency}

Stepping motor is the actuator of electronic cam system. Its output precision affects directly the system motion precision. Stepping motor function is to transform electrical impulse signal into a 
corresponding angular displacement. The angular displacement of stepping motor is proportional to input pulse strictly. Adjust electric pulses frequency of single-chip microcomputer, stepping motor speeds governing [4]. The following is the derivation process and equation of the electrical impulse frequency when system adopts 3-phase reaction stepping motor.

Suppose polynomial movement rule of cam straight moving follower motion as following:

$$
s=\sum_{i=0}^{n} c_{i} \varphi^{i}
$$

In the Eq. 1, $\varphi$ is motion angle of cam, $C_{i}$ is movement coefficient. Obtain first-order derivative of Eq.1 as following:

$$
v_{1}=\sum_{i=1}^{n} i c_{i} \omega_{i} \varphi^{i-1}
$$

In the Eq.2, $\omega_{i}$ is movement angular velocity of cam.

Suppose screw linear velocity is $v$, screw speed is $n_{1}$, thread pitch is $P$, thread line number is $N$ :

$$
v_{2}=\frac{N n_{1} P}{60}
$$

Suppose $v_{1}=v_{2}$, speed of screw is $n_{2}$ :

$$
n_{2}=\frac{60}{P N} \times \sum_{i=1}^{n} i c_{i} \omega_{i} \varphi^{i-1}
$$

Suppose reduction ratio of reducer between stepping motor and screw is ${ }^{i}$, motor speed ${ }^{n^{3}}$ can be written as following:

$$
n_{3}=\frac{60}{P N} \times i \times \sum_{i=1}^{n} i c_{i} \omega_{i} \varphi^{i-1}
$$

Suppose the tooth number of rotor gear of stepping motor is $Z$, the circular pitch is $\tau$ :

$$
\tau=\frac{2 \pi}{Z}
$$

Every electricity makes rotor run one step, step distance angle is $\beta$ as following:

$$
\beta=\frac{2 \pi}{Z k m}
$$

In the Eq.7,K is state coefficient( $\mathrm{K}$ is 1 according to single three clap and double three clap ,K is 2 according to single six clap and double six clap)

Suppose the electrical pulse frequency is $f$, step distance angle is showed with radian, stepping motor speed can be written as following:

$$
n_{3}=\frac{\beta \times f}{2 \pi} \times 60=\frac{\frac{2 \pi}{Z k m} \times f}{2 \pi} \times 60=\frac{60 f}{Z k m}
$$

Unite the Eq.5 with the Eq.8, the equation of electrical pulse frequency $f$ can be written as following:

$$
f=\frac{Z k m}{P N} \times i \times \sum_{i=1}^{n} i c_{i} \omega_{i} \varphi^{i-1}
$$

\section{Summary}

The result of study shows that electronic cam system has the following advantages of mechanical cam mechanism:

1) Cam contour curve is stored in the computer, electronic cam system does not exist wear.

2) When output movement changes, numerical value or setting simply changes without the need to manufacture new cam.

In the future, electronic cam system is used to realize in any movement rules and great stroke 
linear reciprocating motion especially.

\section{References}

[1] Mike, Woelfel. Introduction to electronic cam [J]. Assembly Automation,1999,19(1):17-24. [2] H.Makino. Smart cam application to robot control [J]. Assembly Automation,1999,19(1): 39-46.

[3] Leslie Langlauf. Using electronic cams for motion control [J]. Power Transmission Design, 1996,6:39-41.

[4] Yongquan Yu. The application of Single-chip microcomputer in the control system [M].

Beijing:electronic industry press,2003.

[5] Xingzhong Deng. Electromechanical transmission control [M]. Wuhan:Huazhong university of science and technology press,2001. 